\title{
NOUVELLE
}

\section{Calcium et olfaction chez un insecte modèle : du marqueur d'activité à l'acteur de plasticité}

Jean-Christophe Sandoz, Valérie Raymond-Delpech, Nina Deisig, Monique Gauthier, Martin Giurfa
GDR 2688, Centre de Recherches sur la Cognition Animale, CNRS UMR 5169, Université Paul Sabatier,

118, route de Narbonne, 31062 Toulouse Cedex 04, France. sandoz@cict.fr
> Jusqu'à ces dernières années, le fonctionnement du système nerveux olfactif était mal connu. Les données anatomiques montraient une remarquable conservation des circuits nerveux olfactifs des invertébrés aux vertébrés. Ainsi, le premier relais nerveux olfactif, le bulbe olfactif des vertébrés ou le lobe antennaire des insectes, présente une organisation très semblable en sous-unités anatomiques, les glomérules [1]. Leur nombre, allant d'une quarantaine chez la drosophile à plusieurs milliers chez les mammifères, correspond à peu près au nombre de récepteurs membranaires aux odeurs potentiellement exprimés dans chaque espèce. Enfin, il avait été montré, chez le rat en particulier, que les neurones sensoriels olfactifs exprimant un type donné de récepteur membranaire aux odeurs se projettent dans le même glomérule [2]. On suspectait donc que les glomérules sont à la base de la représentation nerveuse des odeurs mais encore fallait-il le démontrer. Le développement des techniques d'imagerie fonctionnelle a fourni les moyens de le faire [3], bien que nombre de ces techniques (2 désoxyglucose radioactif, c-fos) ne permettaient pas de marquages chez l'animal vivant. C'est donc grâce à l'imagerie calcique, et aux sondes fluorescentes permettant de révéler des variations fines de concentration du $\mathrm{Ca}^{2+}$ intracellulaire dans le cerveau in vivo, que ce champ de la neurobiologie a véritablement pu se développer. C'est aussi, de manière inattendue, chez un invertébré, l'abeille domestique, que cet exploit a été réalisé pour la première fois
[4]. Cette espèce au cerveau simple (Figure $1 A-B, 10^{-3} \mathrm{~cm}^{3}$ et $10^{6}$ neurones contre 1 $400 \mathrm{~cm}^{3}$ et $10^{11}$ neurones pour I'homme) mais néanmoins capable d'exploits cognitifs étonnants, est un modèle phare dans l'étude des bases nerveuses de la perception et de l'apprentissage olfactifs [5].

\section{$\mathrm{Ca}^{2+}$ marqueur d'activité}

Au cours d'une expérience d'imagerie calcique in vivo, l'abeille entière est placée dans une chambre d'enregistrement. La cuticule (non innervée) de sa tête est découpée et le cerveau est découvert. Une sonde fluorescente calcique qui a la propriété de pénétrer dans les cellules ( $\mathrm{Ca}^{2+}$ Green-2AM) est déposée en bain sur le cerveau. Après marquage, la présentation d'une odeur induit une augmentation topique de fluorescence dans le lobe antennaire, au niveau de zones sphériques de 30-50 $\mu \mathrm{m}$ de diamètre (Figure 1C). Des marquages anatomiques montrent que l'activité nerveuse ainsi révélée est localisée au sein des glomérules. Cette technique a permis de démontrer que les odeurs induisent des motifs d'activation glomérulaire spécifiques: à chaque odeur correspond une combinaison particulière de glomérules [4]. Ces motifs d'activité sont reproductibles non seulement chez un même individu, mais aussi d'un individu à l'autre. La morphologie du lobe antennaire étant particulièrement bien conservée, on peut reconnaître chaque glomérule par sa localisation, sa taille ou sa forme chez des individus différents. II a donc été possible de cartographier systématiquement les réponses des glomérules à de nombreuses odeurs classées en fonction de leur formule chimique (fonction aldéhyde, alcool ou cétone, longueur de la chaîne aliphatique) [6]. Ainsi, une similitude de structure chimique entre odeurs se traduit par une superposition des motifs d'activité glomérulaire. II semble donc qu'on ait réussi à décrypter une partie du «code olfactif» dans le cerveau de l'abeille. II subsistait cependant une question importante: à quel point les phénomènes physiologiques enregistrés grâce à l'imagerie calcique correspondent-ils aux «sensations» olfactives de l'abeille? Pour répondre à cette question, nous avons réalisé une expérience d'apprentissage olfactif dont le but était d'établir les critères de similarité entre odeurs au niveau perceptuel [7]. Au laboratoire (Figure IA), les abeilles peuvent apprendre à associer une odeur à un renforcement alimentaire, au cours du conditionnement de l'extension du proboscis (trompe). Cette réponse réflexe est déclenchée normalement par la stimulation des antennes avec de l'eau sucrée. Pour conditionner cette réponse, on présente une odeur (stimulus conditionnel) juste avant le renforcement sucré (stimulus inconditionnel). Après apprentissage, l'odeur apprise, présentée seule, déclenchera I'extension du proboscis. De même, une autre odeur perçue par l'abeille comme similaire à l'odeur apprise, pourra déclencher une réponse, dite de généralisation. Nous avons ainsi mesuré les réponses de 
généralisation de plus de 2000 abeilles mesurant ainsi la similitude perceptuelle parmi 120 paires d'odeurs. La forte corrélation obtenue entre les données comportementales et les données d'imagerie calcique a ainsi prouvé que deux odeurs induisant des motifs d'activation glomérulaire similaires dans le cerveau sont traitées comme similaires par les abeilles dans leur comportement. On a donc bien accès, grâce à l'imagerie calcique in vivo, à une partie du « code olfactif ».

\section{$\mathrm{Ca}^{2+}$ acteur de plasticité}

Le lobe antennaire est une des structures cérébrales impliquées dans la formation de la mémoire olfactive. Des travaux d'imagerie calcique ont montré une plasticité des signaux glomérulaires $[8,9]$. Une de ces études a ainsi comparé les signaux calciques obtenus avant et après un apprentissage olfactif dans lequel une odeur $A$ est récompensée et une odeur B ne l'est pas [8]. Quelques minutes après l'apprentissage, les signaux calciques induits par l'odeur $A$ étaient significativement augmentés, ce qui n'était pas le cas pour l'odeur B. L'importance $\mathrm{du} \mathrm{Ca}^{2+}$ comme acteur précoce dans les phénomènes de plasticité synaptique est bien admise. II peut agir aussi bien au niveau pré-que post-synaptique, et provenir du milieu extracellulaire ou des réserves intracellulaires [10]. Chez les insectes, la formation d'une mémoire olfactive à long terme, dépendante comme chez les vertébrés de la synthèse de nouvelles protéines, peut être induite par une stimulation cal-

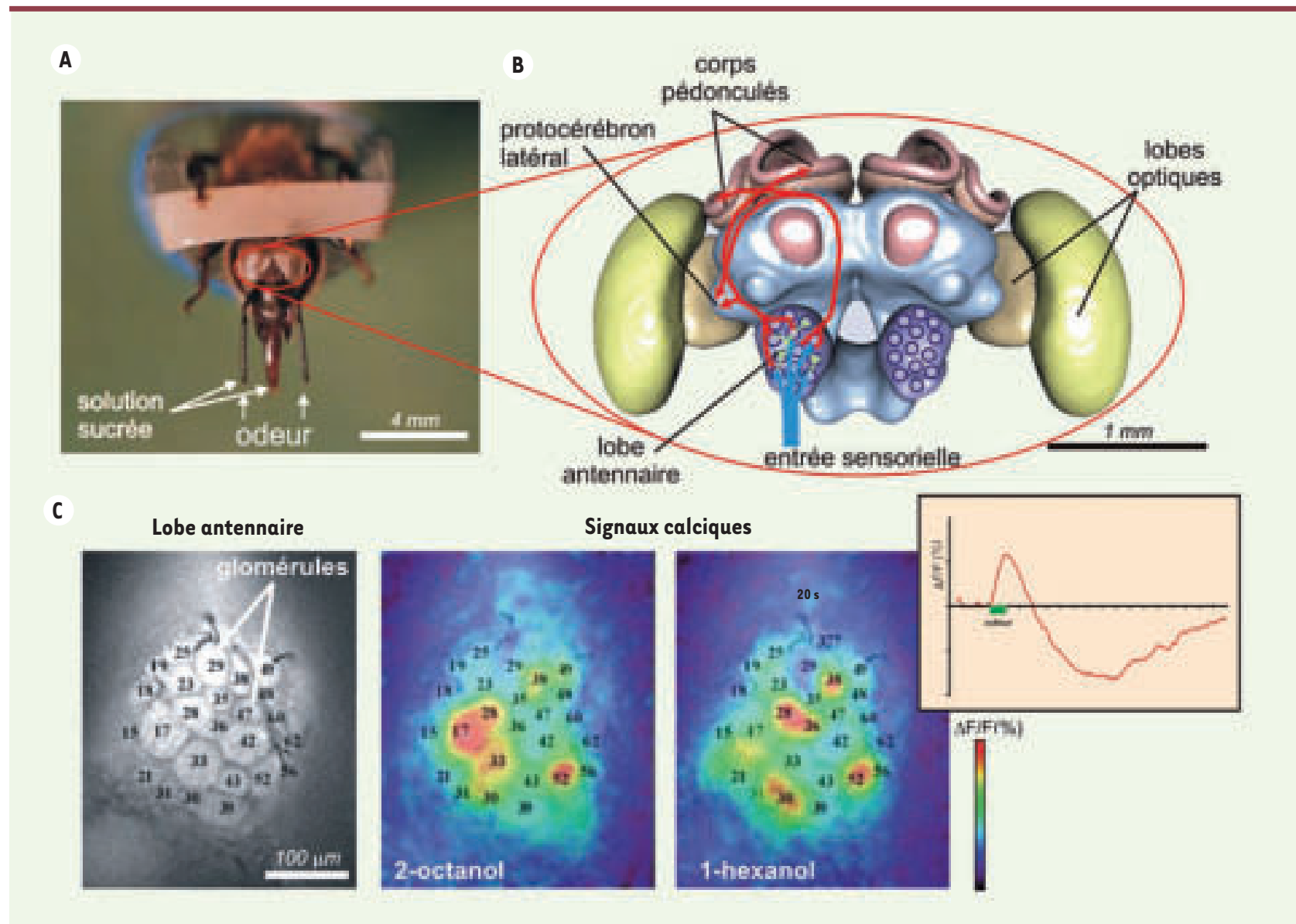

Figure 1. A. Voix nerveuse olfactive de l'abeille domestique, et code olfactif dans le lobe antennaire : abeille en contention lors des expériences de conditionnement pavlovien. Les abeilles apprennent à associer une odeur (présentée aux antennes) à une récompense sucrée (présentée tout d'abord aux antennes, puis au proboscis). La réponse comportementale conditionnée est l'extension du proboscis (trompe). B. Modèle en trois dimensions du cerveau d'abeille (adapté d'après [15]) : les récepteurs olfactifs des antennes envoient l'information (flèche bleue) vers un premier centre nerveux, le lobe antennaire (homologue du bulbe olfactif des vertébrés). Au sein des 160 glomérules, ils forment des contacts synaptiques avec deux autres types cellulaires : des interneurones locaux qui opèrent des inhibitions entre glomérules (flèches vertes), et des neurones extrinsèques, les neurones de projection (flèches rouges). Après traitement par le lobe antennaire, les neurones de projection envoient l'information vers deux centres supérieurs, les corps pédonculés (centre d'intégration reliant plusieurs modalités sensorielles) et le protocérébron latéral (rôle encore obscur). C. Signaux calciques dans le lobe antennaire d'abeille in vivo : le lobe antennaire contient 160 glomérules, dont 30 sont visibles en imagerie et identifiables d'un individu à l'autre. Des odeurs différentes induisent une activité neuronale - et donc une augmentation de la concentration de $\mathrm{Ca}^{2+}$ - dans une combinaison spécifique de glomérules (niveaux d'activation codés en fausses couleurs, du bleu foncé au rouge). Encadré : cinétique des signaux calciques, montrant un signal biphasique, dont le maximum est obtenu 1 seconde après le début de la stimulation olfactive. 
cique. Ainsi, chez le grillon, un traitement induisant une augmentation intracellulaire de $\mathrm{Ca}^{2+}$, associé à une seule association odeur-récompense (qui ne produit normalement qu'une mémoire à court terme) est suffisant pour produire une mémoire à long terme [11].

La connaissance de l'origine des flux calciques révélés par l'imagerie in vivo dans le lobe antennaire $[4,6,8,9]$ est déterminante pour comprendre les phénomènes de perception et d'apprentissage olfactifs. Au sein des glomérules, différents types neuronaux s'associent en réseau. Les neurones sensoriels olfactifs $(\sim 0000)$ se connectent avec des neurones de deuxième ordre, les neurones de projection ( 800), qui transmettent I'information traitée dans le lobe antennaire vers les centres nerveux supérieurs (Figure 1B). Dans les glomérules, des neurones inhibiteurs locaux ( 4 000) se connectent avec les deux autres types cellulaires. De nombreux éléments indiquent que les neurones sensoriels et neurones de projection sont cholinergiques. C'est pourquoi l'acétylcholine (ACh) est présentée comme le neurotransmetteur de la voie nerveuse olfactive. De plus, si l'on bloque la transmission liée à l'ACh à l'aide d'agents pharmacologiques, on observe des troubles de l'apprentissage et de la mémoire olfactive qui indiquent que des récepteurs nicotiniques et muscariniques sont présents, et sont impliqués dans des processus mnésiques différents [12]. Les récepteurs ionotropes nicotiniques (AChRn) sont perméables aux cations et induisent une entrée de $\mathrm{Ca}^{2+}$, soit par le canal ionique, soit du fait de la dépolarisation. Les récepteurs métabotropes muscariniques (AChRm) entraînent l'activation de cascades intracellulaires pouvant conduire à la mobilisation des stocks calciques intracellulaires. On pense que les deux types de récepteurs sont présents dans le lobe antennaire. En particulier, des ARN messagers codant pour des sous-unités entrant dans la composition de différents types de récepteurs nicotiniques ont été révélés par hybridation in situ dans les cellules du lobe antennaire [13]. Lors de la formation de la mémoire à long terme, le $\mathrm{Ca}^{2+}$ libéré par les deux voies citées plus haut, peut agir soit directement sur les facteurs de transcription (par exemple, facteurs DREAM), soit de manière indirecte (par exemple, facteurs CREB), par l'intermédiaire des protéines liant le $\mathrm{Ca}^{2+}$ (calmodulines) et d'autres acteurs intermédiaires. Chez l'abeille, le monoxyde d'azote (NO), l'AMPc et la PKA sont tous impliqués dans la formation de la mémoire olfactive à long terme [14]. Les travaux ultérieurs devront expliquer comment la signalisation calcique au sein des glomérules donne lieu à une mémoire stable et spécifique de l'odeur apprise (donc liée au motif d'activité glomérulaire) et quels sont les gènes dépendant $\mathrm{du} \mathrm{Ca}^{2+}$ qui sont alors exprimés. Le développement de méthodes permettant d'imager sélectivement les différentes populations neuronales du lobe, ou de révéler in vivo les flux des autres acteurs de la plasticité (NO, AMPc, etc.) seront des étapes importantes. Enfin, la relation existant entre les processus mnésiques ayant lieu dans le lobe antennaire et ceux impliquant les centres cérébraux supérieurs, sera aussi analysée. $\diamond$

\section{Calcium and olfaction}

in an insect model: activity marker and actor of plasticity

\section{RÉFÉRENCES}

1. Hildebrand JG, Shepherd GM. Mechanisms of olfactory discrimination: converging evidence for common principles across phyla. Annu Rev Neurosci 1997 ; $18:$ 5999-6008

2. Vassar R, Chao SK, Sitcheran R, et al. Topographic organization of sensory projections to the olfactory bulb. Cell $1994 ; 79$ : 981-91.

3. Rodrigues V. Spatial coding of olfactory information in the antennal lobe of Drosophila melanogaster. Brain Res 1988; 453: 299-307.

4. Joerges J, Kuttner A, Galizia CG, et al. Representations of odours and odour mixtures visualized in the honeybee brain. Nature 1997 ; 387 : 285-8.

5. Menzel R, Giurfa M. Cognitive architecture of a minibrain: the honeybee. Trends Cogn Sci 2001 ; 5 : 62-71.

6. Sachse S, Rappert A, Galizia CG. The spatial representation of chemical structures in the antennal lobe of honeybees: steps towards the olfactory code. EurJ Neurosci 1999; 11 : 3970-82.

7. Guerrieri F, Schubert M, Sandoz JC, et al. Perceptual and neural olfactory similarity in honeybees. PLoS Biol 2005; 3: 1-14.

8. Faber T, Joerges J, Menzel R. Associative learning modifies neural representations of odors in the insect brain. Nat Neurosci $1999 ; 2: 74-8$.

9. Sandoz JC, Galizia CG, Menzel R. Side-specific olfactory conditioning leads to more specific odor representation between sides but not within sides in the honeybee antennal lobes. Neurosci 2003; $120: 1137-48$.

10. Berridge MJ. Neuronal calcium signaling. Neuron 1998 ; $21: 13-26$.

11. Matsumoto $Y$, Unoki S, Aonuma H, et al. Critical role of nitric oxide-cGMP cascade in the formation of cAMPdependent long-term memory. Learn Mem 2006 ; $13: 35-44$

12. Gauthier M, Dacher M, Thany S, et al. Involvement of alpha-bungarotoxin-sensitive nicotinic brain receptors in long-term memory formation in the honeybee (Apis mellifera). Neurobiol Learn Mem 2006 ; 86 : 164-74.

13. Thany SH, Crozatier M, Raymond-Delpech V, et al. G. Apis $\alpha 2$, Apis $\alpha 7-1$ and Apis $\alpha 7-2$ : three new neuronal nicotinic acetylcholine receptor $\alpha$-subunits in the honeybee brain. Gene 2005 ; 344 : 125-32.

14. Müller U. Prolonged activation of cAMP-dependent protein kinase during conditioning induces long-term memory in honeybees. Neuron $2000 ; 27$ : 159-68.

15. Brandt R, Rohlfing T, Rybak J, et al. Three-dimensional average-shape atlas of the honeybee brain and its applications.J Comp Neurol $2005 ; 492: 1-19$.
ILLUSTRATIONS DeS ARTICLES (vignettes): $p$. 53 : structure présumée du canal $P / Q$ comportant les différentes mutations répertoriées, ainsi qu'une trace de ce type de courant calcique. Le fait qu'un canal calcique spécifiquement exprimé dans les neurones soit impliqué dans la migraine hémiplégique familiale a beaucoup frappé les esprits (c) photo Norbert Weiss) - p. 64 : motoneurone (photo Christopher Henderson (C) Photothèque Inserm) - p. 67 : le marqueur lysosomal Lamp-1 est représenté en vert, alors que les complexes CMH de classe I sont en rouge. Les cellules colorées sont des cellules dendritiques humaines, produites par culture de monocytes de sang humain en présence d'IL-4 et de GM-CSF pendant une semaine. La photo montre des cellules non activées, où l'on trouve une bonne partie des molécules du CMH-I dans l'appareil de Golgi, et LAMP-1 dans des structures tubulaires (c) photo Peter van Endert) - p. 75 : ovocyte humain juste après la fécondation (photo Jean Parinaud - (c) Photothèque Inserm) - p. 81, p. 88, p. 93: lésion d'athérome au niveau de la racine de l'aorte (ㄷ photo Giuseppina Caligiuri) - p. 95 : triple hélice d'ADN (photo Sheng Sun-Jian - (C) Photothèque Inserm) - p. 102 : embryon au stade blastocyste (c) photo Jean-Paul Renard) p. 105 : image d'hémophagocytose (@ photo Geneviève de Saint-Basile). 


\section{NOUVELLE}

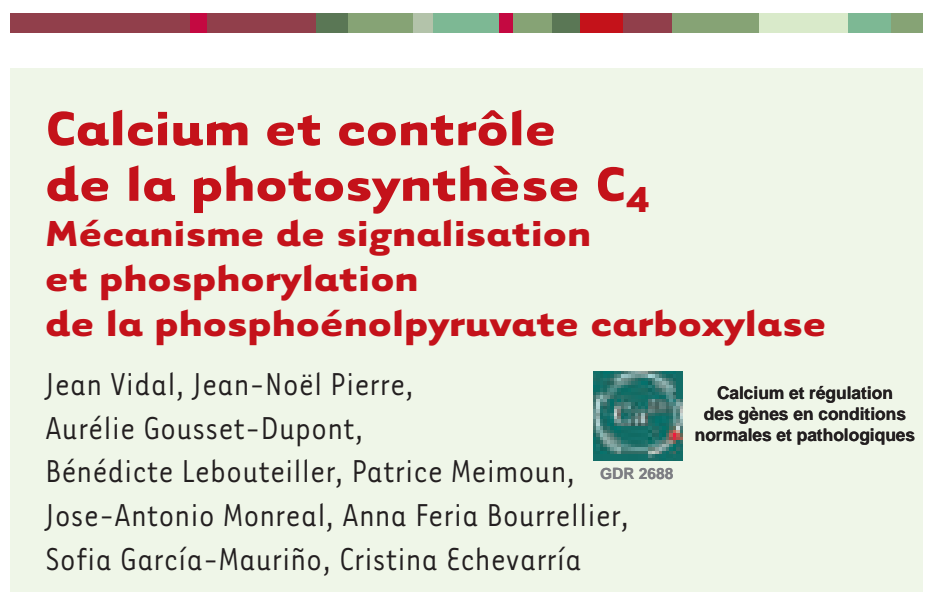

Cadre anatomique

et métabolique de la photosynthèse $C_{4}$ Les plantes de type $C_{4}$ présentent des caractéristiques anatomiques et biochimiques qui composent ce que l'on nomme communément le syndrome $\mathrm{C}_{4}$. L'architecture de la feuille est en couronne (anatomie Kranz), formée de deux strates cellulaires concentriques, le mésophylle et la gaine périvasculaire (Figure l). II existe plusieurs variantes du modèle $C_{4}$ mais le schéma général de division des tâches dans ces deux types cellulaires est conservé. Ainsi, deux cycles, $C_{4}$ et de Benson-Calvin coopèrent à la fonction d'assimilation du $\mathrm{CO}_{2}$ par la feuille (Figure 1). Dans ce contexte photosynthétique fondé sur une séparation physique des enzymes et impliquant un intense trafic intercellulaire de métabolites, la phosphoénolpyruvate carboxylase (PEPC, EC 4.1.1.31) catalyse la première étape de la voie métabolique (carboxylation du PEP par le bicarbonate) dans le mésophylle et fait l'objet d'une régulation particulièrement élaborée.

\section{La phosphorylation de la PEPC} photosynthétique par la PEPC kinase module ses propriétés fonctionnelles La PEPC photosynthétique est un homotétramère de masse $440 \mathrm{kDa}$ dont l'activité est sous contrôle métabolique; le malate (rétro-inhibiteur), le glucose-6-P $(G-6 P)$, les trioses-P ( $T-P)$ et la glycine (activateurs allostériques) en sont les principaux effecteurs. La phosphorylation de l'enzyme à la lumière sur une sérine du domaine amino-terminal de la sousunité (ERHHSIDAQLR) module l'affinité des effecteurs, de sorte qu'une protection accrue contre le malate est observée (par exemple, Vmax et Ki malate augmentent, Ka G-6P diminue) $[1,2]$.

Le clonage de l'ADNc et des gènes de PEPC kinase (PEPCK) a été réalisé récemment à partir de plantes $C_{3}, C_{4}$ et $\operatorname{CAM}[3,4]$, permettant d'établir la structure primaire de l'enzyme et de révéler des caractéristiques originales: (1) sa taille (32 kDa) est celle d'une sous unité catalytique, sans extensions; (2) son activité est contrôlée au niveau transcriptionnel (quantité), processus fortement stimulé par les stress salins ( $\mathrm{NaCl}, \mathrm{LiCl})[5]$, et par des métabolites effecteurs (malate et G-6P) ; (3) elle est phylogénétiquement apparentée aux CDPK (calcium dependent protein kinases, superfamille des (DPK/SnRK), bien qu'elle ne possède pas de site de fixation du calcium: (4) enfin, la seule cible physiologique connue de cette enzyme est la PEPC. L'inhibition de la phosphorylation de la PEPC in vivo lors d'un traitement des feuilles par la cycloheximide (CHX: composé bloquant la synthèse de la PEPCk et l'augmentation de son activité catalytique) [6] provoque une perte presque totale de la fixation photosynthétique de $\mathrm{CO}_{2}$ [7]. Nous avons recherché par quel mécanisme l'activité de
J. Vidal, J.N. Pierre, A. Gousset-Dupont,

B. Lebouteiller, P. Meimoun,

A. Feria Bourrellier :

Institut de Biotechnologie des Plantes, Université de Paris-Sud, UMR CNRS 8618 et GDR 2688, 91405 Orsay Cedex, France.

J.A. Monreal, S. García-Mauriño,

C. Echevarría : Laboratoire de Physiologie

Végétale, Faculté de Biologie,

91405 Orsay Cedex, France.

Jean.vidal@ibp.u-psud.fr

la PEPCk est mise en place à la lumière dans le mésophylle.

\section{L'activité de la PEPCk est contrôlée} par une chaîne de transduction du signal lumineux impliquant les flux calciques dans le mésophylle Une technique d'obtention de protoplastes de cellules de mésophylle (Digitaria sanguinalis et sorgho), et une approche combinant la pharmacologie cellulaire, la microscopie confocale et la cytométrie de flux ont été mises au point pour décrypter le mécanisme de transduction du signal lumineux [8]. Cette démarche expérimentale nous a permis dans un premier temps d'identifier certains relais de la chaîne, notamment et en séquence: (1) un métabolite photosynthétique, l'acide 3-phosphoglycérique (formé dans la gaine périvasculaire par le cycle de Benson-Calvin et transporté dans les chloroplastes des cellules de mésophylle sous forme protonée) provoque l'alcalinisation $\mathrm{du} \mathrm{pH}$ cytosolique $(\mathrm{pHc})$ : (2) un efflux de $\mathrm{Ca}^{2+}$ vacuolaire dû à une phospholipase C (PI$\mathrm{PLC}$ ) et la production transitoire $\mathrm{d}^{\prime} \mathrm{PP}_{3}$; (3) l'activation d'une protéine kinase dépendante du $\mathrm{Ca}^{2+} ;(4)$ la synthèse de la PEPCk, indépendante du $\mathrm{Ca}^{2+}$.

Dans ce mécanisme de transduction, l'implication du calcium n'est qu'inférée des données pharmacologiques. Appliquées aux protoplastes de mésophylle $C_{4}$, les techniques classiques d'image- 